\title{
Design Optimization of Round Strands Made by Twisted Stacks of HTS Tapes
}

\author{
Nikolay Bykovsky, Davide Uglietti, Rainer Wesche, and Pierluigi Bruzzone
}

(Invited Paper)

\begin{abstract}
A twisted stack of high-temperature superconducting (HTS) tapes soldered into copper profiles was used as a strand in the fabrication of the $60-\mathrm{kA} / 12$-T HTS cable prototype at the Center for Research in Plasma Physics (CRPP). Considering the strand in general as a modular element for the high-current cables, design parameters of the strand need to be optimized in order to best fulfill requirements of appropriate application field. During the development program of the HTS fusion cable at CRPP, influence of the design parameters on the strand's performance was obtained as input data for the optimization process. In this paper, we summarize the results of the twisting, bending, and transverse pressure tests on strands of various tapes and profile's geometries. Finite-element model for the transverse pressure test was developed, validated with the test results, and used for the design proposals. Effect of the stack aspect ratio, tape properties, and anisotropy, as well as selection of HTS material for the strand, will be discussed.
\end{abstract}

Index Terms-Finite-element method (FEM) analysis, hightemperature superconducting (HTS) tapes, mechanical load, round strand.

\section{INTRODUCTION}

D URING the last decade, high-temperature superconducting (HTS) materials, such as Bi2212 round wires, Bi2223, and REBCO tapes, have been well developed by various manufacturers and currently represent a very attractive opportunity for use in high-current cables. It allows one to operate at conditions inaccessible for "classical" low-temperature superconducting (LTS) $\left(\mathrm{Nb}_{3} \mathrm{Sn}\right.$ and $\left.\mathrm{NbTi}\right)$ conductors. Depending on the application, such cables should consist of tens to hundreds of tapes or wires. This necessitates developing cabling concepts, which are essentially new for the thin tape geometry not available before with LTS. Several successful cabling concepts were proposed by various laboratories, with their own weak and strong points [1]-[3]. For example, the twisted stacked HTS tapes conductor (TSTC) [1] provides high current density,

Manuscript received September 17, 2015; revised November 10, 2015 and December 18, 2015; accepted December 18, 2015. Date of publication January 12, 2016; date of current version February 8, 2016. This paper was recommended by Editor L. Chiesa.

The authors are with the Center for Research in Plasma Physics (CRPP), Fusion Technology, Swiss Federal Institute of Technology in Lausanne (EPFL), 5232 Villigen PSI, Switzerland (e-mail: nikolay.bykovsky@psi.ch).

Color versions of one or more of the figures in this paper are available online at http://ieeexplore.ieee.org.

Digital Object Identifier 10.1109/TASC.2016.2517187

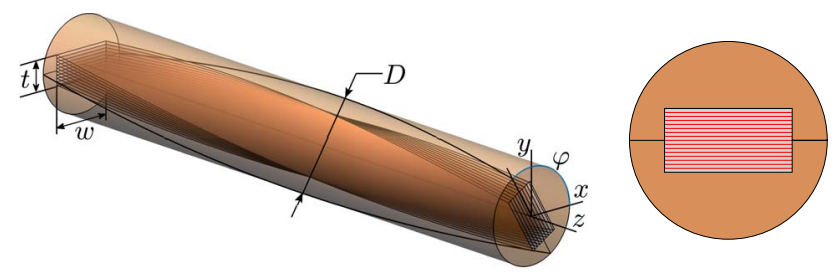

Fig. 1. Design of the strand. (Left) 3-D view. (Right) Cross section.

TABLE I

PARAMETERS OF THE STRAND DESIGN

\begin{tabular}{ll}
\hline Symbol & Description \\
\hline$w$ & $\begin{array}{l}\text { Width of the strand's slot } \\
\text { (width of the tape) }\end{array}$ \\
$t$ & Height of the strand's slot \\
$n$ & Number of tapes in the strand \\
$h$ & Twist-pitch of the strand \\
$D$ & Diameter of the strand \\
\hline
\end{tabular}

efficient material use, and flexibility of the conductor. Noncomplete transposition of the tapes in TSTC concept (inductance of the tape depends on its position in the stack) can lead to a nonuniform current distribution between tapes in ac or transient operating regimes. First high-field-low-temperature tests of the TSTC conductor showed a necessity of additional mechanical reinforcement against the acting Lorentz force [4].

In order to improve current redistribution between tapes in the stack and to provide rigidity to the conductor, at the Center for Research in Plasma Physics (CRPP), an additional encasing of the stack between two copper profiles and soldering the components together were added to the original concept [5]. The proposed design and set of five parameters, which fully describes the geometry, are given in Fig. 1 and Table I. Similarly to the terminology of LTS conductors, we call the proposed element design in Fig. 1 as a "strand."

High-field-low-temperature tests were successfully performed at Karlsruhe Institute of Technology (KIT) with the nontwisted SuperPower 15-tape strand and twisted at $h=$ $320 \mathrm{~mm}$ SuperOx 16-tape strand. Measured performance of both samples is in good agreement with expectations based on the tape's properties. At $12 \mathrm{~T}, 4.2 \mathrm{~K}$, the critical current for both samples is around $3 \mathrm{kA}$. Degradation of the strands' performance during the cycling test was not observed. 
Use of copper shells and soldering sufficiently improves mechanical stability of the stack, but the main negative consequences are reduced ability to the bending and, possibly, twisting. Additionally, the intertape resistance between tapes is significantly lowered by soldering, which provides a uniform current distribution on one hand, but on the other hand, intertape coupling current loss is increased as well. Since the improvement of one property may lead to the worsening of the other, an "ideal" design and manufacturing sequence of the strand are considered as the most balanced for the chosen application field.

In that sense, influence of the design parameters and variations in the manufacturing on the performance of the strand was investigated during the development program of the HTS fusion cable at CRPP. In this paper, we summarize the results of the various electromechanical tests with strands of different tapes' width and geometries of the profiles (see Section II). Concluding recommendations are given in Section III.

\section{Mechanical Properties of the Strand}

\section{A. Twisting}

Twisting of the single tape and the stack of tapes was studied in details in [1]. Expression for the axial strain along the tape's cross section in the stack of tapes is written as

$$
\varepsilon_{\text {twisting }}=\frac{1}{2}\left(\frac{2 \pi}{h}\right)^{2}\left(u^{2}+v^{2}\right)-\varepsilon_{0}
$$

where $(u, v)$ is the coordinate system of the cross section, $u$ is along the wide side of the tape: $-w / 2 \leqslant u \leqslant w / 2$; and $\varepsilon_{0}$ is the relaxation strain representing "sliding effect" during the twisting: tapes have a mechanical ability to let the cross section be free of a longitudinal force. Mathematically, this effect corresponds to the following condition:

$$
F_{\text {axial }}=\int_{S} E(u, v) \varepsilon_{\text {twisting }}(u, v) d u d v=0
$$

where $E$ is the Young's modulus, and $S$ is the cross section of the relaxation region. It is important to point out that, during the twisting, nonsoldered stack behaves the same as a single tape in the axial strain approach since the relaxation region is each individual tape in the stack. Appropriate relaxation strain can be written as $\varepsilon_{0}=1 / 2(2 \pi / h)^{2}\left(w^{2} / 12+v_{k}^{2}\right)$, where $v_{k}$ is the vertical position of the $k$ th tape in the stack.

When soldering is used, there are two options for the manufacture of the stack or the strand: first twist, then solder (TS) or first solder, then twist (ST) [5]. The TS case corresponds to the situation discussed earlier for the nonsoldered stack twisting, whereas the ST case has undesirable effect of a strain accumulation. The stack is considered in the calculation as a combination of two materials, i.e., HTS tapes and solder, whereas for the strand, the copper profiles represent a third domain. Using the axial strain approach, relaxation strains for

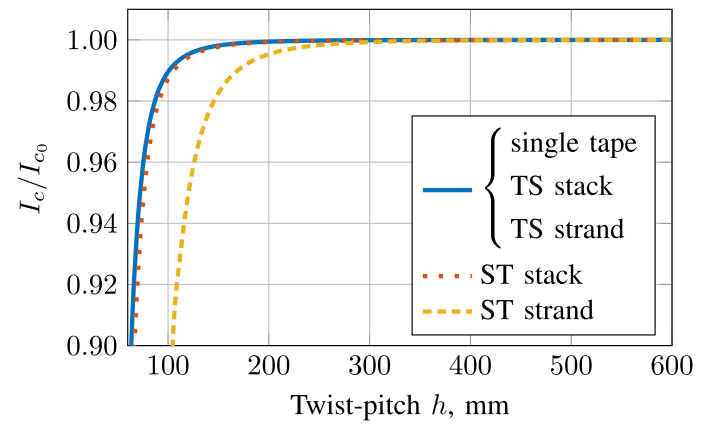

Fig. 2. Calculated $I_{c}$ degradation of the twisted single tape, TS stack, TS strand, ST stack, and ST strand due to the axial strain at $w=4 \mathrm{~mm}, t=2 \mathrm{~mm}$, $n=13, D=6.2 \mathrm{~mm}$.

ST stack and ST strand can be written from (1) and (2) as follows:

$$
\begin{aligned}
\varepsilon_{0}^{\mathrm{ST} \text { stack }} & =\frac{E_{1} I_{1}+E_{2} I_{2}}{E_{1} S_{1}+E_{2} S_{2}} \\
\varepsilon_{0}^{\mathrm{ST} \text { strand }} & =\frac{E_{1} I_{1}+E_{2} I_{2}+E_{3} I_{3}}{E_{1} S_{1}+E_{2} S_{2}+E_{3} S_{3}} \\
I_{j} & =\frac{1}{2}\left(\frac{2 \pi}{h}\right)^{2} \int_{S_{j}}\left(u^{2}+v^{2}\right) d u d v
\end{aligned}
$$

where $S_{j}$ is the cross section of the $j$ th material: $1-\mathrm{HTS}$ tapes; 2 -solder; 3 -copper profiles. Young's modules for the materials at $77 \mathrm{~K}$ are chosen as $E_{1}=157 \mathrm{GPa}$ [6], $E_{2}=$ $45 \mathrm{GPa}$ [7], and $E_{3}=137 \mathrm{GPa}$ [8].

With an available dependence of critical current density over the axial strain $j_{c}(\varepsilon)$, finally, one can calculate degradation of the critical current $I_{c}$. As there is no variation of the strains along the stack's length, a parallel connection of superconductors with various $j_{c}$ can be considered, i.e.,

$$
\frac{I_{c}(h)}{I_{c_{0}}}=\frac{1}{n} \sum_{k=1}^{n} \frac{1}{w} \int_{-w / 2}^{w / 2} \frac{j_{c}\left(\varepsilon_{\text {twisting }}\left(u, v_{k}\right)\right)}{j_{c_{0}}} d u .
$$

The $I_{c} / I_{c_{0}}$ curves of the single tape, TS stack, TS strand, ST stack, and ST strand $(w=4 \mathrm{~mm}, t=2 \mathrm{~mm}, n=13$, $D=6.2 \mathrm{~mm}$ ) made of SuperPower tapes as a function of the twist pitch $h$ are given in Fig. 2. Note that, in the axial strain approach, the curves for the single tape, TS stack, and TS strand are identical. Calculated degradation of the twisted single tape is in good agreement with measured results (for example, see [1]). Increased axial strains in the ST stack and ST strand lead to the earlier degradation of the critical current.

Degradation of the critical current of the ST stack and strand was studied experimentally as well. Thirteen-tape (SuperPower SCS4050) straight soldered stack and strand were prepared for the twisting test. Before assembling each sample, five tapes were measured individually. These tapes were located at positions $1,4,7,10$, and 13 in the stack. The test results are given in Fig. 3 together with the verified dependence of the single tape. Stronger influence of the twist pitch $h$ was obtained comparing with the calculated results, particularly in case of ST strand.

The five initially measured tapes were carefully extracted from the stack and strand at the end of the tests (at around 10\% 


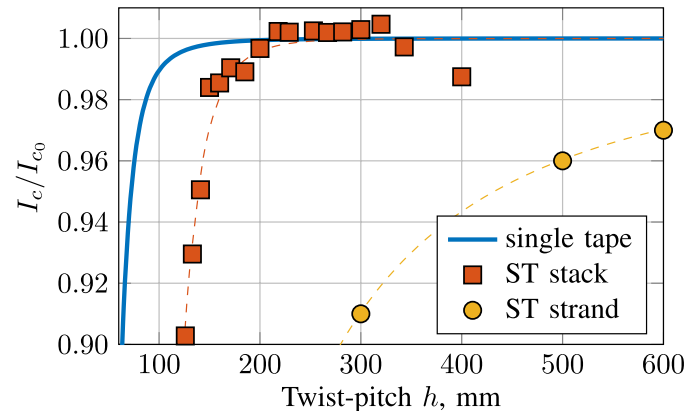

Fig. 3. Measured $I_{c}$ degradation of the twisted 13-tape ST stack and ST strand (77 K, self-field).

of $I_{c}$ degradation). In both cases, the same $10 \%$ degradation was obtained for the central tape (at position 7), whereas the degradation of the outer tapes ( 1 and 13) was the lowest: from $2 \%$ to $6 \%$.

Shear strain is another important component of the strain during the twisting. While the minimum and the maximum of the axial strain are at the center and the corners of the stack, respectively [according to the strain distribution (1)], shear strain components $\gamma_{x z}$ and $\gamma_{y z}$ reach their extremums at the middles of the outer sides of the stack [9]. Unfortunately, even with a known distribution of the shear strain in the stack, there is no appropriate dependence of the critical current density (first attempts were done at KIT [10]). Effect of the shear strain could be at the origin of the difference between the measured and calculated $I_{c}$ degradation of the ST stack and ST strand. In addition, $\gamma_{y z}$ component acting in the tape's plane has the highest impact on the central tape of the stack, which can explain the highest measured degradation on tape 7 in the stack.

As a short conclusion, TS is the favorable manufacturing sequence, allowing to produce the strand with the twist pitch limited only by the properties of the single tape. In the region of low $I_{c}$ degradation $(\lesssim 1 \%)$, critical twist pitch $h_{c}$ at a chosen criteria is proportional to the tape's width $w$, which was obtained from the variation of $w$ in the calculation of $I_{c}$ degradation. For instance, $0.1 \% I_{c}$ degradation corresponds to $h_{c} \approx 40 w$ in the TS approach. As a confirmation, several TS strands presented in the following chapters have no mechanical degradation at twist pitches down to $300 \mathrm{~mm}$.

\section{B. Bending}

Bending of the strands typically occurs during cable manufacturing (due to the transposition requirement) and in a coil winding. Expression for the total axial strain in the twisted strand bent at fixed radius $R$ was obtained in [11] and is written as

$$
\begin{aligned}
\varepsilon_{\text {total }} & =\varepsilon_{\text {twisting }}+\varepsilon_{\text {bending }} \\
\varepsilon_{\text {bending }} & =\frac{u \cos \left(\frac{2 \pi}{h} s\right)-v \sin \left(\frac{2 \pi}{h} s\right)}{R}
\end{aligned}
$$

where $s$ is the arc length of the strand's centerline (longitudinal coordinate); $\varepsilon_{\text {twisting }}$ was discussed earlier (see Section II-A) and used here in TS approach.

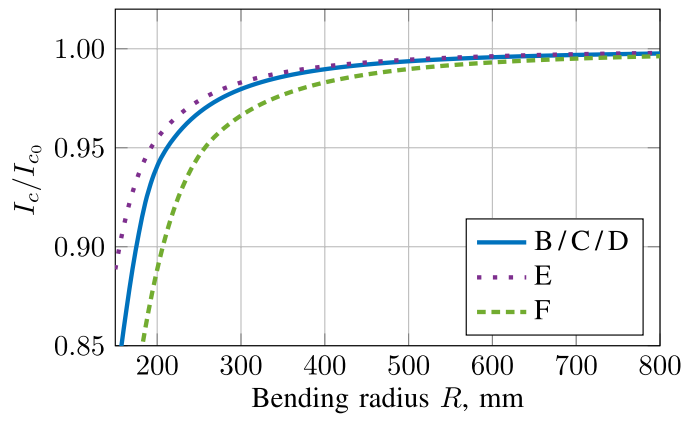

Fig. 4. Calculated $I_{c}$ degradation of bent twisted strands (see Table II for the abbreviations' definition).

Following the distribution of the total strain, the critical current density $j_{c}\left(\varepsilon_{\text {total }}\right)$ changes along the width and length of the tape. As a consequence, calculation of the critical current of the bent twisted strand must deal with both parallel and serial connections of superconducting elements. If the given strand's length $l$ divided into $N_{s}$ elements and for each one the critical current of the cross section $I_{c_{i}}$ is obtained according to (4), then the average critical current of the strand $I_{C}$ is a solution for $N_{s}$ serial-connected superconducting regions problem

$U_{\text {total }}=\sum_{i=1}^{N_{s}} l_{i} E_{c}\left(\frac{I}{I_{c_{i}}}\right)^{n_{i}}=l E_{c}\left(\frac{I}{I_{c}}\right)^{n} \stackrel{I=I_{c}}{\Longrightarrow} \sum_{i=1}^{N_{s}} \frac{l_{i}}{l}\left(\frac{I_{c}}{I_{c_{i}}}\right)^{n_{i}}=1$

where $U_{\text {total }}$ is the total voltage over the chosen strand's length $l, l_{i}$ is the element's length (normally $l_{i}=l / N_{s}$ ), and $E_{c}=$ $1 \mu \mathrm{V} / \mathrm{cm}$. Equation (6) additionally requires a dependence of $n$-value over the axial strain or, as a result, over the critical current. It was assumed simply as $n_{i}=n_{0}^{I_{c_{i}} / I_{c_{0}}}$, which represents a lowering trend for $n$-value during $I_{c}$ degradation. $n_{0}$ was set to 25 , a typical $n$-value for REBCO tapes at $77 \mathrm{~K}$. Variation of $n_{0}$ has an insignificant influence in the calculation.

Results of the bending model for various sets of geometrical parameters are given in Fig. 4. The lines correspond to sets of parameters of SuperPower samples prepared for the measurements. In total, eight types of TS strands were used in the bending test: they are presented in Table II. $I_{c}$ degradation of each strand as a function of bending radius $R$ is given in Fig. 5. Good agreement between calculation and test results was obtained, which allows one to consider the axial strain as a dominant factor of the degradation during the bending.

The following are several aspects of the strand's manufacturing procedure and geometry that were studied in the bending test.

1) Preliminary annealing of the copper profiles: A versus B. Effectively softened copper profiles by the annealing $\left(1 \mathrm{~h}\right.$ at $\left.300{ }^{\circ} \mathrm{C}\right)$ provide improved bending properties of the strand. The profiles with a lowered yield strength ensure less stress concentration; thus, the influence of the encasing copper on the bending properties is minimized.

2) Geometry of the copper profiles: $\mathrm{B}$ versus $\mathrm{C}$ versus $\mathrm{D}$. The new geometries $(C, D)$ reinforce the soldered connection between copper profiles. As a result, no cracks between copper profiles of the C and D samples were observed, 
TABLE II

TS STRANDS USED IN THE BENDING TEST

\begin{tabular}{|c|c|c|c|c|c|c|c|c|}
\hline Abbreviation & A & B & $\mathrm{C}$ & $\mathrm{D}$ & $\mathrm{E}$ & $\mathrm{F}$ & G & $\mathrm{H}$ \\
\hline Tape's manufacturer & & & - Super & ower - & & 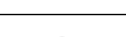 & SuperOx & Sumitomo \\
\hline \multicolumn{9}{|l|}{ Cross section } \\
\hline Tape's width $w, \mathrm{~mm}$ & 4 & 4 & 4 & 4 & 3 & 4 & 4 & 4.3 \\
\hline Diameter $D, \mathrm{~mm}$ & 6.2 & 6.2 & 7 & 7 & 6 & 8 & 6.2 & 8 \\
\hline Number of tapes $n$ & 15 & 15 & 15 & 15 & 26 & 32 & 15 & 13 \\
\hline Twist-pitch $h, \mathrm{~mm}$ & 300 & 300 & 300 & 300 & 240 & 340 & 300 & 400 \\
\hline Profile annealing & No & Yes & Yes & Yes & Yes & Yes & Yes & Yes \\
\hline$j_{e}{ }^{a}, \mathrm{~A} / \mathrm{mm}^{2}$ & 94 & 94 & 74 & 74 & 131 & 121 & 90 & 114 \\
\hline Copper area, $\mathrm{mm}^{2}$ & $25(81 \%)$ & $25(81 \%)$ & $33(85 \%)$ & $33(85 \%)$ & $22(79 \%)$ & $39(78 \%)$ & $25(81 \%)$ & $33(66 \%)$ \\
\hline$R_{c}^{b}, \mathrm{~mm}$ & 420 & 180 & 190 & 190 & 205 & 360 & 275 & 920 \\
\hline
\end{tabular}

${ }^{a}$ Engineering current density at $(12 \mathrm{~T}, 4.2 \mathrm{~K}) ;{ }^{b}$ Critical bending radius @ $5 \% I_{c}$ loss

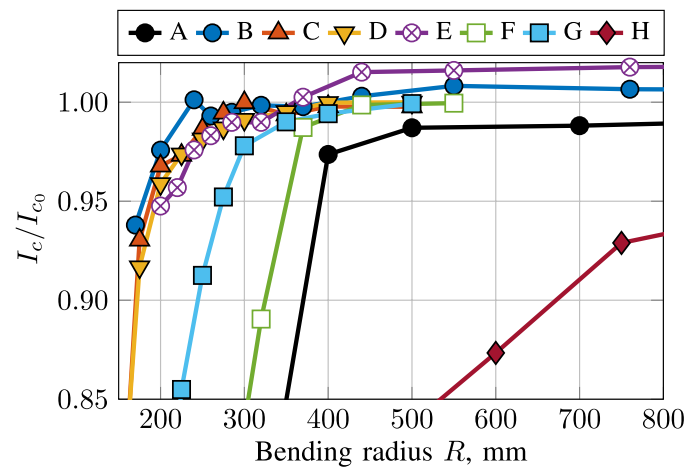

Fig. 5. Measured $I_{c}$ degradation of bent twisted strands (77 K, self-field; see Table II for the abbreviations' definition).

whereas for the B sample, opening of the profiles happened at around $240 \mathrm{~mm}$. Nevertheless, the relatively complicated fabrication procedure of the D sample and the reduced current density of both the $\mathrm{C}$ and $\mathrm{D}$ samples make them less attractive. Higher $j_{e}$ can be achieved with $\mathrm{C}$ and $\mathrm{D}$ samples by reducing the diameter, which was chosen as $7 \mathrm{~mm}$ only due to the easier fabrication at the laboratory scale.

3) Effect of the stack's aspect ratio: C versus F. Current density of the F sample is sufficiently increased, but at the same time, the critical bending radius $R_{c}$ is almost twice as large compared with the $\mathrm{C}$ sample. A compromised solution is needed in this case.

4) Effect of the tape's width w: E versus F. Both the E and F samples have a square stack, the only difference is the tape's width (although the twist pitch for both samples is different, it was chosen conservatively - an influence on the bending properties is not expected). While the current density of the two samples is comparable and noticeably higher than the other samples, the bending properties of the E sample is much better due to the narrower tapes. Currently, the E-type geometry is the most balanced and favorable in electromechanical terms.

5) Manufacturer of REBCO tapes: B versus $G$ obtained difference between B (SuperPower) and G (SuperOx) samples is in agreement with a recently published comparison of mechanical properties of various REBCO coated conductors [12]. While the $R_{c}$ of SuperOx strand is higher, it is still applicable for construction of relevant high-current cable based on such strands.

6) REBCO versus Bi2223 tapes: The $\mathrm{F}$ versus $\mathrm{H}$ sample, manufactured with $1 \mathrm{G}$ Sumutomo H-type tapes, is the weakest one in terms of bending with $R_{c}=920 \mathrm{~mm}$. That value, representing relatively low mechanical properties of $1 \mathrm{G}$ tapes itself, is too high to use such strand in a construction of high-current cables; thus, other geometries must be considered.

\section{Transverse Pressure}

A strand in the cable is subjected to the transverse mechanical load due to the acting Lorentz force. This is a typical "plane strain" problem of continuum mechanics. With the assumption over several strain components $\varepsilon_{z}=\gamma_{x z}=\gamma_{y z}=0$, inplane stress-strain elastic relation simplifies to the following form [13]:

$$
\left[\begin{array}{c}
\sigma_{x} \\
\sigma_{y} \\
\tau_{x y}
\end{array}\right]=\frac{E}{(1+\nu)(1-2 \nu)}\left(\begin{array}{ccc}
1-\nu & \nu & 0 \\
\nu & 1-\nu & 0 \\
0 & 0 & 1 / 2-\nu
\end{array}\right)\left[\begin{array}{c}
\varepsilon_{x} \\
\varepsilon_{y} \\
\gamma_{x y}
\end{array}\right]
$$

where $\left(\sigma_{x}, \sigma_{y}, \tau_{x y}\right)$ and $\left(\varepsilon_{x}, \varepsilon_{y}, \gamma_{x y}\right)$ are stress and strain components, respectively; and $E$ and $\nu$ are the Young's modulus and the Poisson's ratio of the appropriate material domain, respectively. The strand is simply considered as a combination of two domains: copper (c-domain) and "solid" stack of tapes (s-domain). Young's modulus of s-domain was obtained according to the mixture rule: $E_{s}=E_{\text {tape }} n d_{t} / t+E_{\text {solder }}(1-$ $\left.n d_{t} / t\right)$, where $d_{t}$ is the thickness of the tape, $E_{\text {tape }}=157 \mathrm{GPa}$ [6], and $E_{\text {solder }}=45 \mathrm{GPa}$ [7].

Three cases corresponding to the geometry of the A, G, and E samples (see Table II) were studied in the plane strain finiteelement method (FEM) model using MATLAB PDE Toolbox. Mechanical parameters used for these cases are given in Table III.

The solution of the problem is a distribution of horizontal and vertical displacements $(u(x, y), v(x, y))$. Stress and strain 
TABLE III

Mechanical Parameters Used in the Model (77 K)

\begin{tabular}{lccc}
\hline Parameter & $\mathrm{A}$ & $\mathrm{G}$ & $\mathrm{E}$ \\
\hline$E_{c}, \mathrm{GPa}$ & 137 & 80 & 80 \\
$E_{s}, \mathrm{GPa}$ & 129 & 129 & 142 \\
$\nu_{c}$ & 0.34 & 0.34 & 0.34 \\
$\nu_{s}$ & 0.3 & 0.3 & 0.3 \\
\hline
\end{tabular}
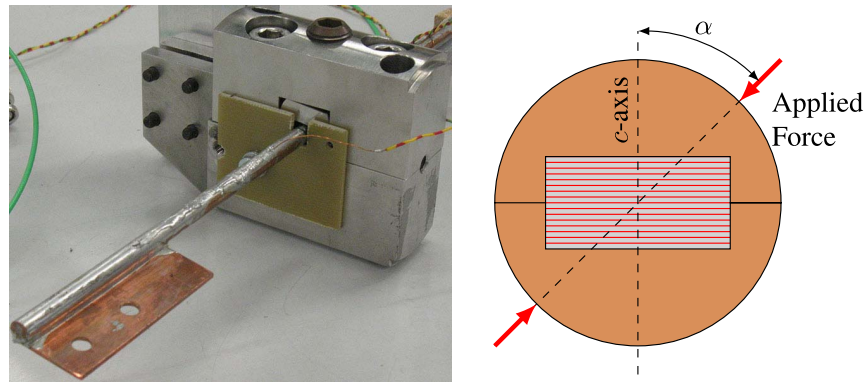

Fig. 6. (Left) Experimental setup. (Right) Definition of angle of orientation $\alpha$.

components are spatial derivatives of the displacements over particular coordinate. In order to obtain $I_{c}$ degradation of the strand, $j_{c}$ dependencies over the parallel and perpendicular (to the wide side of tape) strains of the single tape are needed, but not available. Thus, considering only the mechanical part of the problem, in-plane principal stresses $\left(\sigma_{1}, \sigma_{2}\right)$ and longitudinal one $\sigma_{3}=\nu\left(\sigma_{1}+\sigma_{2}\right)$ were combined in a single effective measure-von Mises stress $\sigma_{v}$-which has the following definition in the plane strain case:

$$
\sigma_{v}=\sqrt{\left(\sigma_{1}^{2}+\sigma_{2}^{2}\right)\left(\nu^{2}-\nu+1\right)+\sigma_{1} \sigma_{2}\left(2 \nu^{2}-2 \nu-1\right)} .
$$

The von Mises stress is typically used to define the yield criterion as $\sigma_{v}=\sigma_{\text {yield. }}$. For the current needs, $\sigma_{v}$ will be used only to have a scalar description of the mechanical problem.

The model is considered only in the elastic regime of materials, which means that the problem is linear. In other words, only relative distribution of stresses is important, whereas absolute values are proportional to the external applied pressure. Thus, all the following results of the calculations will be normalized to the midplane pressure, which is defined as an applied force $F$ per area of the strand's midplane $l D: p_{\text {midplane }}=F /(l D)$. This parameter represents a vertical stress in the strand's midplane in case of uniformly distributed solution. Thereby, normalization to the $p_{\text {midplane }}$ is a measure of the deviation of real nonuniform solution from the uniform one.

By changing the angle between the $c$-axis and the direction of the applied force (see Fig. 6), the anisotropy of the strand's mechanical properties was studied. Three values of $\alpha$ were analyzed in the FEM model and measurements: $0^{\circ}$ (least loaded case), $60^{\circ}$ (potentially dangerous case due to the possible shear breakage), and $90^{\circ}$ (most loaded case).

As an example, a solution of the model in terms of normalized von Mises stress for the geometry of the E sample (see Table II) is given in Fig. 7. Focusing the attention on the stack's domain, a statistical approach was applied to the

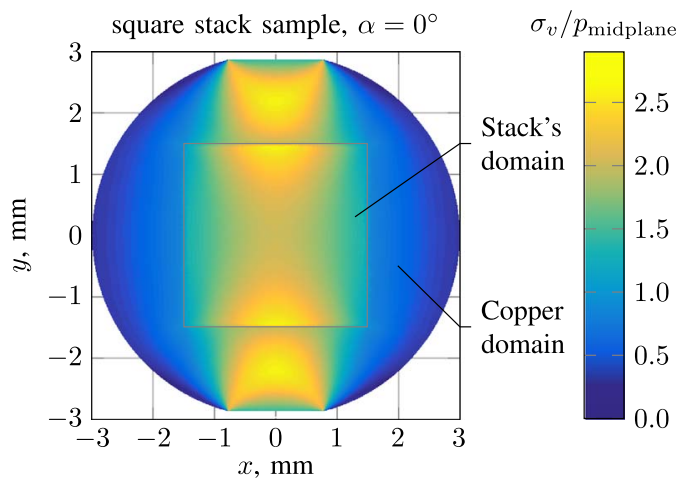

Fig. 7. Distribution of the von Mises stress $\sigma_{v}$ for the geometry of the E sample (see Table II).

distribution of the von Mises stress in order to have a more obvious interpretation and comparison of the model's results (see [14] for the full details). The main idea of the approach is to build a cumulative area function (CAF) for the s-domain (9). At the fixed $\sigma_{v}$, CAF gives a relative area of the stack which is subjected to the von Mises stress less than $\sigma_{v}$ (similar to the definition of cumulative distribution function in statistics), i.e.,

$$
\operatorname{CAF}\left(\sigma_{v}\right)=\frac{1}{S} \int_{\sigma_{v}^{\prime}<\sigma_{v}} d S^{\prime} .
$$

CAF is presented in Fig. 8 for the geometries of the A, G, and E samples at three fixed angles of orientation $\alpha$. Both the A and $\mathrm{G}$ cases show a high mechanical anisotropy, i.e., the curves at $0^{\circ}, 60^{\circ}$, and $90^{\circ}$ are far apart, whereas this is not the case for the $\mathrm{E}$ case (note that the $\mathrm{E}$ case results at $0^{\circ}$ and $90^{\circ}$ are identical). Tails at high $\sigma_{v} / p_{\text {midplane }}$ ratio of the yellow $\left(\alpha=90^{\circ}\right)$ and red $\left(\alpha=60^{\circ}\right)$ curves indicate a presence of dangerous regions in the s-domain, which can be at the origin of the strand's breakage. The shift to the right of the $\mathrm{G}$ sample curves relatively to the A sample curves is an effect of the copper profile's annealing: softer profiles lead to the higher stress in the stack.

An average von Mises stress $\left\langle\sigma_{v}\right\rangle$ over the area of the s-domain $S$ was obtained as follows:

$$
\left\langle\sigma_{v}\right\rangle=\frac{1}{S} \int_{S} \sigma_{v} d S=\int_{0}^{\sigma_{v_{\max }}}\left(1-\operatorname{CAF}\left(\sigma_{v}\right)\right) d \sigma_{v} .
$$

$\left\langle\sigma_{v}\right\rangle$ as a function of the angle of orientation $\alpha$ is given in Fig. 9: the annealing of the copper profiles (A versus $G$ ) increases the stress in the s-domain (but also improves the bending properties of the strand; see previous section); modified geometry of the copper profiles ( $G$ versus E) eliminates anisotropy of the mechanical properties. As long as the twisted geometry of the strand will result in all possible angles of orientation in the cable, reduction of the maximal average von Mises stress $\left\langle\sigma_{v}\right\rangle$ is the main benefit, which was implemented with E sample.

Experimental study of the strand's $I_{c}$ degradation under the transverse pressure was performed on the three samples manufactured according to the parameters' set of A, G, and E samples in Table II, with the difference that the A sample was nontwisted, whereas the $G$ and $E$ samples were twisted at 


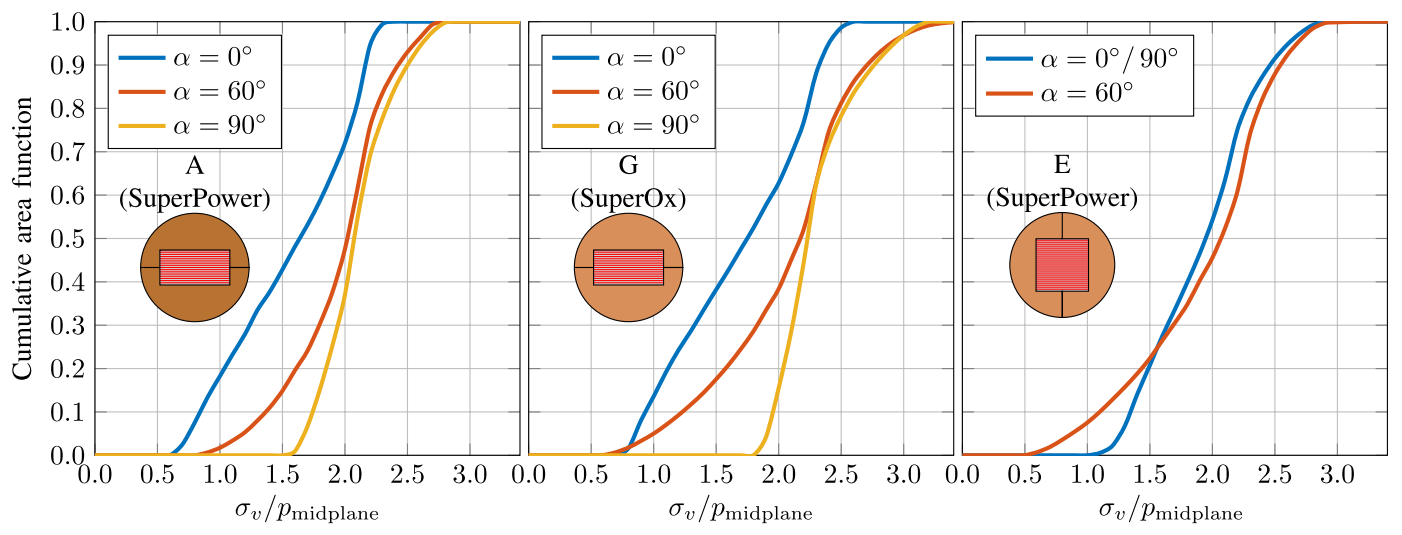

Fig. 8. CAF of the s-domain of the A, G, and E samples (see Table II) as a function of normalized von Mises stress at various angles of orientation $\alpha$.

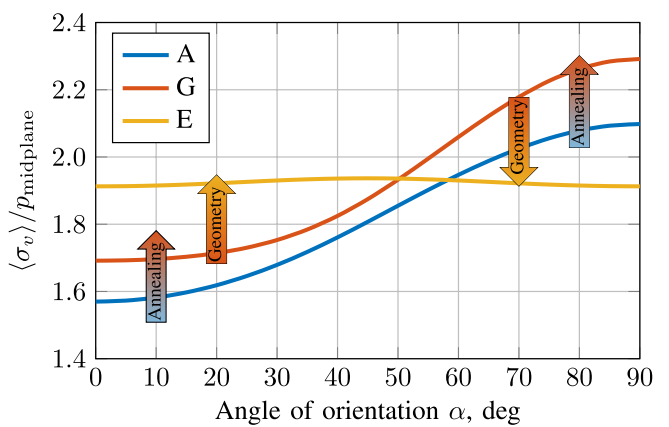

Fig. 9. Angular dependence of the normalized average von Mises stress.

$h=300 \mathrm{~mm}$ and $h=280 \mathrm{~mm}$, respectively. Twisting of the samples allows changing $\alpha$ in the test simply by longitudinal shift, whereas in nontwisted case, the strand must be rotated. Short regions of each sample, corresponding to the $0^{\circ}, 60^{\circ}$, and $90^{\circ}$ orientations (variation due to the twisting is negligible), were subjected to the transverse force in a liquid nitrogen bath. The force was applied by tightening the bolt, which transfers the load to the steel anvil located on top of the strand (see Fig. 6). A piezoelectric sensor, which is located at the bottom of the strand, was used to measure the applied force. The midplane pressure $p_{\text {midplane }}$ is obtained as the measured force divided by the width of the anvil and the diameter of the strand.

A summary of the test results is presented in Fig. 10. Measured $I_{c}$ degradation of the three strands qualitatively follows the results of the FEM model discussed earlier. The A and $\mathrm{G}$ samples are very tolerant to the applied force at $0^{\circ}$ (up to $110 \mathrm{MPa}$ ). At $60^{\circ}$, both samples have an abrupt $I_{c}$ degradation in the region of $60 \mathrm{MPa}$, which corresponds to fracture effects in the strand (may result in cracks between copper profiles). Finally, $I_{c}$ degradation of these samples at $90^{\circ}$ sets the operational limits: $25 \mathrm{MPa}$ for A and $20 \mathrm{MPa}$ for G. Due to a slightly weaker mechanical performance of the tapes made by SuperOx compared with those by SuperPower [12], the B sample could have higher operational limit than the G one (see Table II). In contrast with the A and $\mathrm{G}$ samples, measured curves for the E sample are very similar at $0^{\circ}$ and $90^{\circ}$, whereas the earliest degradation was obtained at $60^{\circ}$. Fracture effects were not observed in this case. Operational limit can be set up as $35 \mathrm{MPa}$.
If the strands are used in the Rutherford-type cable representing a flat two-layered assembly of strands (see Fig. 11), midplane pressure can be estimated as $p_{\text {midplane }} \approx 2 I B / D$. The strand carrying $2.5 \mathrm{kA}$ at $12 \mathrm{~T}$ will be subjected to roughly $10 \mathrm{MPa}$, making the proposed strand design appropriate for this cable option.

\section{SUMMARY AND OUTLOOK}

Summarizing the experimental and numerical results over the various mechanical and electrical properties of the strand presented in this paper, the following concluding recommendations can be formulated.

1) Soldering of the strand components (copper profiles and HTS tapes) must be performed after the twisting, with the preliminary annealed copper profiles. This allows one to eliminate strain accumulation effect and lower the limits for the twist pitch and bending radius.

2) Use of narrower tape is desirable as it introduces only positive changes: increases current density of the strand, reduces the hysteresis loss, and lowers the mechanical limits for the twist pitch $h$ and bending radius $R$.

3) Optimal geometry of the copper profile is the one with the split line parallel to the $c$-axis of tapes in the stack (for instance, see geometry of C sample in Table II). This provides the most balanced strand's properties against the acting transverse pressure.

Currently, the E-type strand is considered as the most promising one for the construction of the 50-kA/12-T cable prototype. Work on an appropriate cable design will start soon. Future cable prototypes will be tested in the European Dipole (EDIPO) test facility at CRPP, including $I_{c}, T_{c s}$, ac loss, and currentcycling measurements at various operating conditions.

As promised by the manufacturers of the coated conductors, thinner tapes, based on 30- $\mu \mathrm{m}$ hastelloy layer instead of $50 \mu \mathrm{m}$, may come soon to the market. If the geometrical changes will not affect any other properties of the tape, an E-type strand containing around 35 tapes could carry up to $5 \mathrm{kA}$ at $12 \mathrm{~T}$ and $4.2 \mathrm{~K}$. This may significantly reduce the number of the strands required for the cable fabrication. 


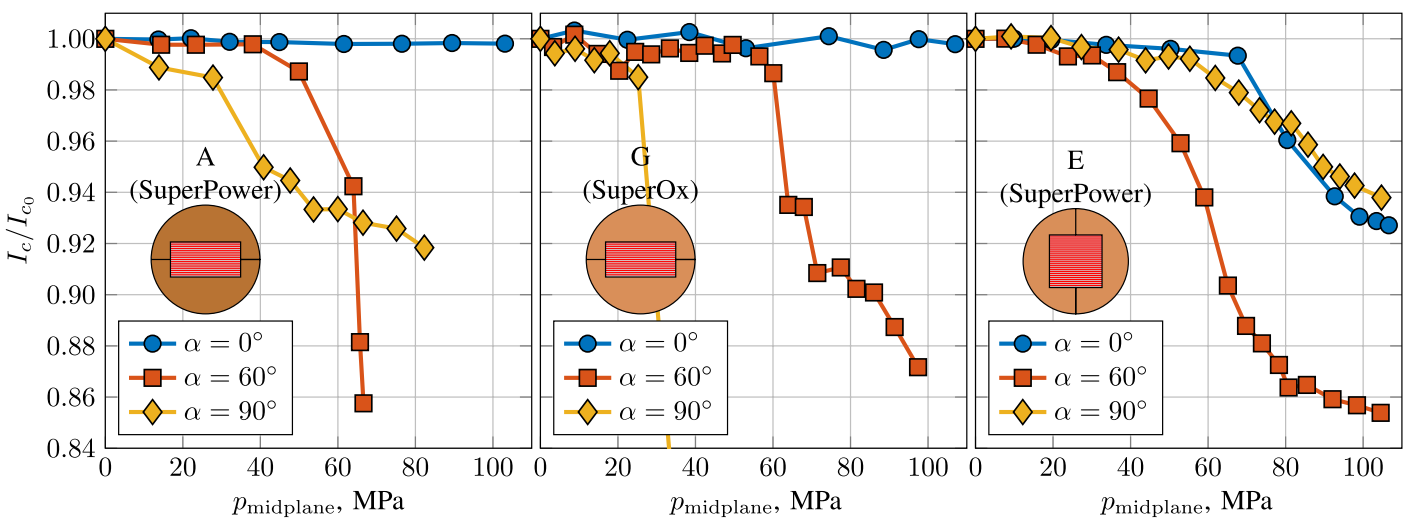

Fig. 10. Measured $I_{c}$ degradation of the A, G, and $\mathrm{E}$ samples (see Table II) as a function of $p_{\text {midplane }}$ at various angles of orientation $\alpha$ (77 K, self-field).

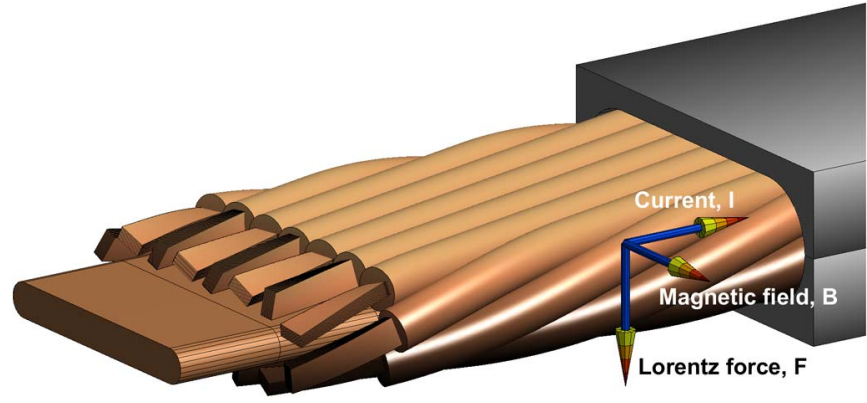

Fig. 11. Cored Rutherford cable design. Directions of the transport current, external magnetic field, and Lorentz force are also shown.

\section{ACKNOWLEDGMENT}

The authors would like to thank the Paul Scherrer Institut for the technical support. N. Bykovsky would like to thank C. Calzolaio for the useful discussions about the transverse pressure FEM modeling.

\section{REFERENCES}

[1] M. Takayasu, L. Chiesa, L. Bromberg, and J. V. Minervini, "HTS twisted stacked-tape cable conductor," Supercond. Sci. Technol., vol. 25, no. 1, Jan. 2012, Art. ID 014011.

[2] W. Goldacker et al., "Status of high transport current ROEBEL assembled coated conductor cables," Supercond. Sci. Technol., vol. 22, no. 3, Mar. 2009, Art. ID 034003.

[3] D. C. van der Laan, X. F. Lu, and L. F. Goodrich, "Compact $\mathrm{GdBa}_{2} \mathrm{Cu}_{3} \mathrm{O}_{7-\delta}$ coated conductor cables for electric power transmission and magnet applications," Supercond. Sci. Technol., vol. 24, no. 4, Apr. 2011, Art. ID 042001.

[4] C. Barth et al., "Temperature- and field dependent characterization of a twisted stacked-tape cable," Supercond. Sci. Technol., vol. 28, no. 4, Apr. 2015, Art. ID 045015 .

[5] D. Uglietti, R. Wesche, and P. Bruzzone, "Fabrication trials of round strands composed of coated conductor tapes," IEEE Trans. Appl. Supercond., vol. 23, no. 3, Jun. 2013, Art. ID 4802104.

[6] K. Ilin et al., "Experiments and FE modeling of stress-strain state in ReBCO tape under tensile, torsional and transverse load," Supercond. Sci. Technol., vol. 28, no. 5, May 2015, Art. ID 055006.

[7] C. Basaran and J. Jiang, "Measuring intrinsic elastic modulus of $\mathrm{Pb} / \mathrm{Sn}$ solder alloys," Mech. Mater., vol. 34, no. 6, pp. 349-362, Jun. 2002.

[8] R. P. Reed and A. F. Clark, Eds., Materials at Low Temperatures. Metals Park, OH, USA: American Society for Metals, 1983, 590 pp.

[9] K.-P. Weiss, W. Goldacker, and M. Nannini, "Finite element analysis of torsion experiments on HTSC tapes," IEEE Trans. Appl. Supercond., vol. 21, no. 3, pp. 3102-3106, Jun. 2011.

[10] C. Barth, K.-P. Weiss, and W. Goldacker, "Influence of shear stress on current carrying capabilities of high temperature superconductor tapes," IEEE Trans. Appl. Supercond, vol. 21, no. 3, pp. 3098-3101, Jun. 2011.

[11] N. Bykovsky, D. Uglietti, R. Wesche, and P. Bruzzone, "Strain management in HTS high current cables," IEEE Trans. Appl. Supercond., vol. 25, no. 3, Jun. 2015, Art. ID 4800304.
[12] C. Barth, G. Mondonico, and C. Senatore, "Electro-mechanical properties of REBCO coated conductors from various industrial manufacturers at 77 K, self-field and 4.2 K, 19 T," Supercond. Sci. Technol., vol. 28, no. 4, Feb. 2015, Art. ID 045011

[13] S. Timoshenko and J. N. Goodier, Theory of Elasticity. New York, NY USA: McGraw-Hill, 1970.

[14] C. Calzolaio et al., "Electro-mechanical properties of PIT $\mathrm{Nb}_{3} \mathrm{Sn}$ wires under transverse stress: Experimental results and FEM analysis," Supercond. Sci. Technol., vol. 28, no. 5, May 2015, Art. ID 055014.

Nikolay Bykovsky was born in Moscow, Russia, on June 6, 1990. He received the master's degree in physics and engineering from the National Research Nuclear University MEPhI (Moscow Engineering Physics Institute), Moscow, in 2013. He is currently working toward the Ph.D. degree in physics from the Swiss Plasma Center, École Polytechnique Fédérale de Lausanne, Villigen, Switzerland.

From 2012 to 2013, he was an Engineer with the Russian Scientific R\&D Cable Institute, Moscow, working in the area of applied superconductivity. Since 2013, he has been with the Swiss Plasma Center, École Polytechnique Fédérale de Lausanne, working on his Ph.D. thesis in physics titled "HTS high current cable for fusion application."

Davide Uglietti was born in Galliate, Italy, on June 11, 1972. He received the M.S. degree in physics from Universitegli Studi di Milano, Milan, Italy, in 1997, and the Ph.D. degree in physics from the University of Geneva, Geneva, Switzerland, in 2006, working on microstructure and transport properties of Nb3Sn.

From 1998 to 2000, he was with Pirelli Cables and Systems, Milan, working on Bi2223 power transmission cables. From 2007 to 2010, he was with the National Institute of Material Science, Tsukuba, Japan, where he was engaged on the development of coated conductor insert coils. Since 2010, he has been a Scientist with the Swiss Plasma Center, École Polytechnique Fédérale de Lausanne, Villigen, Switzerland.

Rainer Wesche was born in Überlingen, Germany, on October 16, 1956. He received the M.S. degree in physics and the Ph.D. degree from the University of Konstanz, Konstanz, Germany, in 1984 and 1988, respectively.

From 1985 to 1989, he was an Assistant with the University of Konstanz. In 1989, he joined the Paul Scherrer Institute, Villigen, Switzerland, where he led an experimental study of high-current applications of high-Tc superconductors. Since 1994, he has been a Research Scientist with École Polytechnique Fédérale de Lausanne, Villigen. His current research is in the field of applied superconductivity.

Pierluigi Bruzzone was born in Genoa, Italy, on November 9, 1955. He received the degree in physics from the University of Genoa, Genoa, Italy, in 1979, and the Ph.D. degree in physics from ETH Zurich, Zurich, Switzerland, in 1987, working on ac losses on high-current superconducting cables.

He has been with the National Research Council of Italy (CNR) and the Swiss Institute for Nuclear Physics (SIN). Then, he joined ABB, Oerlikon, working in the superconductivity department. In 1990, he joined the NET Team in Garching and later the Superconducting Coils and Structure Division of the ITER Project Central Team in Naka, Japan. Since 1997, he has been with the Swiss Plasma Center, École Polytechnique Fédérale de Lausanne, Villigen, Switzerland, where he has lead the superconductivity section since 2001. 